\title{
A note of caution on the Waterloo-Stanford Group Scale of Hypnotic Susceptibility: A Brief
}

Communication ${ }^{1}$

Etzel CARDEÑA

Devin B. TERHUNE ${ }^{2}$

Department of Psychology, Lund University, Lund, Sweden

${ }^{1}$ This study was supported by grant 54/06 from the Bial Foundation

${ }^{2}$ Address correspondence to Etzel Cardeña, PhD, Thorsen Professor, Department of Psychology, Center for Research on Consciousness and Anomalous Psychology (CERCAP), Lund University, P.O. Box 213 SE-221 00, Lund, Sweden. E-mail: Etzel. Cardena@psychology.lu.se

Abstract: The scant literature on the sequelae of hypnotizability- measurement instruments indicates that different scales produce different reactions. The Waterloo-Stanford Group Scale of Hypnotic Susceptibility, Form C (WSGC) was developed as a group version of the Stanford Hypnotic Susceptibility Scale, Form C (SHSS:C). Parallel to the literature showing that the SHSS:C can produce more negative aftereffects than the Harvard Group Scale of Hypnotic Susceptibility, Form A, we report preliminary descriptive statistics documenting the infrequent occurrence of negative reactions to the WSGC age-regression item. We recommend that experimenters take this matter into consid- eration and, at the very least, ensure the presence of a clinician with hypnotic experience during the administration of the WSGC. 
The impact of hypnotizability screening measures and hypnosis experiments on participants is an understudied topic. A book on the subject estimated that around $22 \%$ of participants in hypnosis experiments suffer complications, comparing favorably to an estimate of $35 \%$ from stage hypnosis (MacHovec, 1986, p. 14). An influential study helped put matters into perspective. Coe and Ryken (1979) compared the aftereffects of receiving the Stanford Hypnotic Susceptibility Scale, Form C (SHSS:C; Weitzenhoffer \& Hilgard, 1962) with four typical college events: (a) participating in a brief verbal learning experiment, (b) taking an exam, (c) attending a class, and (d) college life in general $(N=70)$. They found that hypnosis did not produce more negative effects than the other tasks (taking an exam was rated as the most stressful) and was rated overall as the most pleasant activity. A more careful reading of the data shows, however, that although no one gave an overall rating of hypnosis as unpleasant or very unpleasant, $49 \%$ of participants mentioned negative sequelae (Coe \& Ryken, 1979, p. 677). This contrasts with another study that found $31 \%$ of participants reporting such experiences after the SHSS:C (Hilgard, 1974). Apprehension and anxiety by a minority of participants about the testing may actually precede the hypnotic experience (Laurence \& Labelle, 1989).

There is evidence that the type of scale employed has an impact. Crawford, Hilgard, and Macdonald (1982) observed that while 5\% of participants reported minor transient negative experiences following the Harvard Group Scale of Hypnotic Susceptibility, Form A (HGSHS; Shor \& Orne, 1962), 29\% reported them after the SHSS:C. Crawford et al. also reported specifically that cognitive distortions or confusion were associated with passing the cognitive items in the SHSS:C; although they did not address which specific items were most problematic.

Although it is the most widely used group measure of hypnotizability, the HGSHS has some limitations, foremost its association with acqui- escence and the lack of more difficult cognitive items, which may not allow a sensitive classification of very highly hypnotizable individuals (Council, 1999; Register \& Kihlstrom, 1986). The Waterloo-Stanford Group Scale of Hypnotic Susceptibility, Form C (WSGC; Bowers, 1993, 1998) was developed to alleviate these limitations. This scale is a group adaptation of the SHSS:C, which is often considered the gold standard of individual hypnotic tests (Council). Given the 
evidence that the SHSS:C produces considerably more negative reactions than the HGSHS, it is reasonable to ask whether the WSGC has similar effects on participants. We are unaware of any systematic research of this point and present anecdotal evidence here, which we nonetheless con- sider important for anyone intending to use the WSGC.

The first author has administered the HGSHS for about 20 years to many hundreds of students in the United States and Sweden. Although he has encountered the occasional mild negative reaction, typically a person not feeling quite out of hypnosis, at no point had he witnessed strong emotional reactions to this scale. That changed when we started using the WSGC. We have observed in an $N=642$ of under- graduate students 13 negative reactions, three of them of strong emo- tions and uncontrolled movements that prevented participants from continuing with the scale, eight just of crying, one of a participant not feeling completely awake, and one of a participant getting nauseous during the induction. In 11 of these cases, the observed reactions appear to have occurred in response to the age regression item, which triggered distressing memories or thoughts about unpleasant events (being in a war zone, being bullied, childhood sexual abuse). This $2 \%$ of problems corresponds to the estimated $2 \%$ of intense negative reaction in a research setting (MacHovec, 1986). Nonetheless, the intense distress of some of these reactions (at least one of them lingered for some days) raises an important ethical consideration concerning the use of the WSGC, even if the reactions have been mostly short-lived. We have always had at least one person with clinical training and experience present during screening sessions, so we have been able to deal with these negative responses, which abated after discussion with the clini- cian and additional hypnotic techniques as needed.

The author of the WSGC recommended not using this scale as the first exposure to hypnosis, "perhaps increasing the risk of distress to the more difficult and challenging suggestions" (Bowers, 1993, p. 44); this admonition being based on it not having introductory information and caveats on hypnosis. In our case, even though we have used it as a first test, it has been with students who had read about hypnosis beforehand and received a brief introduction to the topic. We currently emphasize that volunteers are free not to participate and can stop at any time, at which point we can escort them out of the room. And 
provide two alternative grades for each of the two sets of grades in the age-regression items, but we have still encountered negative reactions. Responses from those who have had strong sequelae indicate that the regression item may bring about untoward consequences. One authority in the field of hypnotizability measurement (Erik Woody, personal communication, January 7, 2008) confirmed that he had occasionally encountered crying in response to the age-regression item because that time had not been a happy one in the person's life. A second expert (Michael Nash, personal communication, January 29, 2008) wrote that his lab routinely omits the age-regression item because of the negative responses it may generate.

Besides the potentially distressing nature of the age-regression item, the WSGC may be more problematic than the SHSS:C for two other reasons. The first is that because of the group setting the hypnotist may not be able to detect the first signs of a negative response and cannot shape his or her instructions accordingly; this would especially hold for group hypnosis sessions that utilize audio recordings of the scale administration. The second is that there is evidence that high hypnotiz- ables are more likely to have negative transient sequelae (Laurence \& Labelle, 1989) and also to adopt the emotions of others (Cardeña, Terhune, Lööf, \& Buratti, 2008). One of the three strongest reactions we observed occurred when a participant heard a second one crying during hypnosis and started panicking herself.

The WSGC is a very useful hypnotizability instrument, but we want to emphasize that it requires more precautions than the HGSHS. We suggest that any researchers using the WSGC consider these issues carefully, inquire about and be alert for potentially intense negative reactions and follow them up as necessary, and not administer the scale without a clinician present.

\section{References}

Bowers, K. S. (1993). The Waterloo-Stanford Group C (WSGC) Scale of Hypnotic Susceptibility: Normative and comparative data. International Journal of Clinical and Experimental Hypnosis, 41, $35-46$.

Bowers, K. S. (1998). Waterloo-Stanford Group Scale of Hypnotic Susceptibility, Form C: Manual and 
response booklet. International Journal of Clinical and Experimental Hypnosis, 46, 250-268.

Cardeña, E., Terhune, D., Lööf, A., \& Buratti, S. (2008). Hypnotic experience is related to emotional contagion. International Journal of Clinical and Experimental Hypnosis, 57, 33-46.

Coe, W., \& Ryken, K. (1979). Hypnosis and risks to human subjects. American Psychologist, 34, 673681.

Council, J. R. (1999). Measures of hypnotic responding. In I. Kirsch, A. Capafons, E. Cardeña-Buelna, \& S. Amigó (Eds.), Clinical hypnosis and self-regulation therapy: A cognitive-behavioral perspective (pp. 119-140). Washington, DC: American Psychological Association.

Crawford, H. J., Hilgard, J. R., \& Macdonald, H. (1982). Transient experiences following hypnotic testing and special termination procedures. International Journal of Clinical and Experimental Hypnosis, 30, $117-126$.

Hilgard, J. (1974). Sequelae to hypnosis. International Journal of Clinical and Experimental Hypnosis, $22,281-298$.

Laurence, J.-R., \& Labelle, 1. (1989, August). Positive and negative transient experiences in hypnosis. Paper presented at the 97th Annual Convention of the American Psychological Association, New Orleans, LA.

MacHovec, F. (1986). Hypnosis complications: Prevention and risk management. Springfield, IL: Charles C. Thomas.

Register, P., \& Kihlstrom, J. F. (1986). Finding the hypnotic virtuoso. International Journal of Clinical and Experimental Hypnosis, 34, 84-97.

Shor, R. E., \& Orne, E. C. (1962). Harvard Group Scale of Hypnotic Susceptibility. Palo Alto, CA: Consulting Psychologists Press.

Weitzenhoffer, A. M., \& Hilgard, E. R. (1962). Stanford Hypnotic Susceptibility. Scale, Form C. Palo Alto, CA: Consulting Psychologists Press. 